\title{
A Frequency-Shift based CMOS Magnetic Biosensor with Spatially Uniform Sensor Transducer Gain
}

\author{
Hua Wang, Constantine Sideris, and Ali Hajimiri \\ Department of Electrical Engineering, \\ California Institute of Technology, Pasadena, CA, 91125, USA
}

\begin{abstract}
This paper presents a scalable and ultrasensitive magnetic biosensing scheme based on on-chip LC resonance frequency-shifting. The sensor transducer gain is demonstrated as being location-dependent on the sensing surface and proportional to the local polarization magnetic field strength $|B|^{2}$ generated by the sensing inductor. To improve the gain uniformity, a bowl-shape stacked coil together with floating shimming metal is proposed for the inductor design. As an implementation example, a 16-cell sensor array is designed in a $45 \mathrm{~nm}$ CMOS process. The spatially uniform sensor gain of the array is verified by testing micron-size magnetic particles randomly placed on the sensing surface. The Correlated-DoubleCounting (CDC) noise cancellation scheme is also implemented in the presented design, which achieves a noise suppression of $10.6 \mathrm{~dB}$ with no power overhead. Overall, the presented sensor demonstrates a dynamic range of at least $85.4 \mathrm{~dB}$.
\end{abstract}

\section{INTRODUCTION}

Future Point-of-Care (PoC) molecular level detection requires advanced sensing platforms with hand-held portability, high-sensitivity, low-cost, and battery-level power consumption to replace existing lab-based diagnostics facilities. These PoC systems, once fully developed, can function as mass-deployable units to address on-site medical diagnostic applications such as home-based health care, epidemic disease control, and environmental monitoring.

Although widely used, fluorescence-based molecular detection schemes require bulky and expensive optical devices and experience signal decaying or quenching issues. Magnetic-particle based sensing platforms have been proposed to augment or replace the optical approach. However, magnetic sensors reported so far demand external biasing magnetic fields and/or complicated post-processing, limiting their form factor and cost [1-3]. An ultrasensitive frequencyshift magnetic sensing scheme is proposed as a promising candidate for PoC applications in order to address these issues [4]. In this paper, the sensor transducer gain is first modeled. A new sensing inductor design method is then proposed to engineer the polarization magnetic field and achieve uniform transducer gain, which significantly improves the system dynamic range.

The paper is organized as follows: Section II introduces the frequency-shift magnetic sensing scheme and models the physical transducer gain. Section III presents a design method to achieve a spatially uniform gain response. Based on the proposed design method, a CMOS implementation example is demonstrated together with the CDC noise cancellation scheme in section IV. The measurement results are presented in section $\mathrm{V}$ and VI, which verify the noise suppression functionality and characterize the spatially uniform gain.

\section{SENSOR MECHANIMS AND SENSOR TRANSDUCER GAIN MODELING}

Magnetic biosensors typically adopt sandwich-based bioassays, such as the Enzyme-Linked ImmunoSorbent Assay (ELISA), with magnetic particles used as sensing tags. During the detection process, the pre-deposited molecular probes first capture the target molecules in the sample. The biochemically functionalized magnetic particles are then introduced and immobilized by the captured target molecules. Therefore, by sensing the magnetic particles on the sensor surface, one can directly measure the presence of the target molecules in the test sample both qualitatively and quantitatively.

Our proposed scheme utilizes the on-chip LC resonant tank of an oscillator as the sensor core. The magnetic field generated by the inductor polarizes the magnetic particles present close to the sensor surface, resulting in an increase in total magnetic energy in the space. This leads to an effective increase in the inductance, which translates to a corresponding down-shift in the oscillation frequency [4], shown in Fig.1.

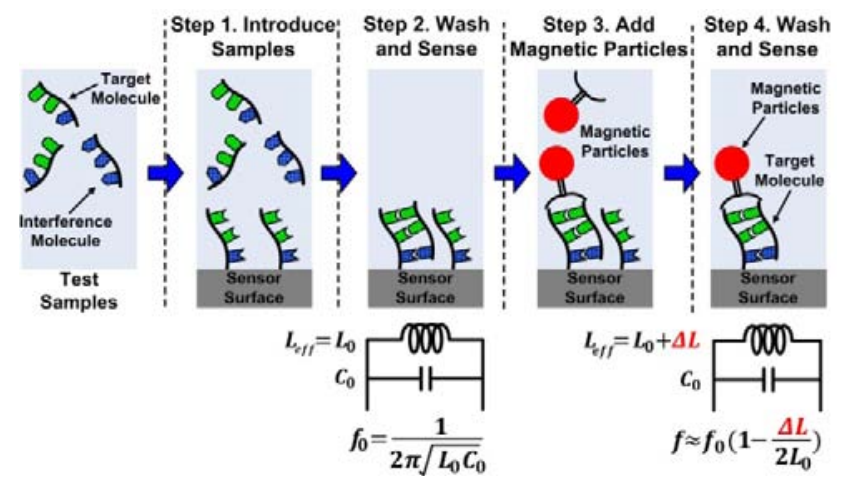

Fig. 1. Frequency-shift magnetic sensing scheme.

The physical process, which relates the presence of magnetic particles with frequency down-shift can be further modeled as follows. Assume a magnetic particle with effective susceptibility $\chi_{\text {eff }}$ and a volume $V_{p}$ is placed close to the onchip sensing inductor. When a current $I$ conducts through the coil, the local polarization magnetic field is $\vec{H}$. Assuming the presence of the magnetic particle will not alter this $\vec{H}$, the total magnetic energy therefore increases by $\Delta E_{m}$ after placing the particle,

$$
\Delta E_{m}=\left(E_{m^{\prime}}-E_{m}\right)=\frac{1}{2} \iiint \vec{H} \cdot \overrightarrow{B^{\prime}} d v-\frac{1}{2} \iiint \vec{H} \cdot \vec{B} d v
$$




$$
\begin{aligned}
& =\frac{\mu_{0}}{2} \iiint_{V_{p}}\left[\|\vec{H}\|^{2}\left(1+\chi_{e f f}\right)-\|\vec{H}\|^{2}\right] d v=\frac{\chi_{e f f}}{2 \mu_{0}} \iiint_{V_{p}}\|\vec{B}\|^{2} d v \\
& \approx \frac{\chi_{e f f}}{2 \mu_{0}}\|\vec{B}\|^{2} V_{p},
\end{aligned}
$$

where $\overrightarrow{B^{\prime}}$ and $\vec{B}$ are the local magnetic flux density with and without the magnetic particle. The approximation holds when the particle is small enough so that the polarization field is homogenous across its volume.

The sensor transducer gain can be defined as the relative frequency-shift (due to the inductance change) per particle as,

$$
\begin{gathered}
\text { Transducer Gain }=\left(\frac{\Delta f}{f_{0}}\right)_{\text {per particle }}=-\frac{\Delta L}{2 L_{0}}=-\frac{1}{2} \cdot \frac{2 \Delta E_{m} / I^{2}}{L_{0}} \\
=-\frac{1}{2} \cdot \frac{2 \frac{\chi_{\text {eff }}}{2 \mu_{0}}\|\vec{B}\|^{2} V_{p} / I^{2}}{L_{0}}=-\frac{1}{2} \cdot \frac{\chi_{e f f} V_{p}}{\mu_{0} L_{0}} \cdot \frac{\|\vec{B}\|^{2}}{I^{2}} .
\end{gathered}
$$

Equation (2) shows that the sensor transducer gain is location-dependent on the sensor surface and is proportional to the field quantity $\|\vec{B}\|^{2} / I^{2}$.

\section{SENSING INDUCTOR DESIGN FOR SPATIALLY UNIFORM SENSOR GAIN}

This location-dependent transducer gain degrades the sensor performance directly. For practical magnetic molecular diagnosis, the positions of the immobilized magnetic particles are distributed randomly on the sensor surface. Although a large number of particles can spatially "average" out this inhomogeneity, the non-uniform transducer gain still results in inconsistent output signals for different particle distributions when detecting small particle counts (at low target molecule concentrations). This creates as an effective noise floor, which may completely mask the fundamental sensor electrical noise- floor (1/f $\mathrm{f}^{3}$ phase noise dependent) [5] and significantly compromises the system's dynamic range, shown in Fig.2.

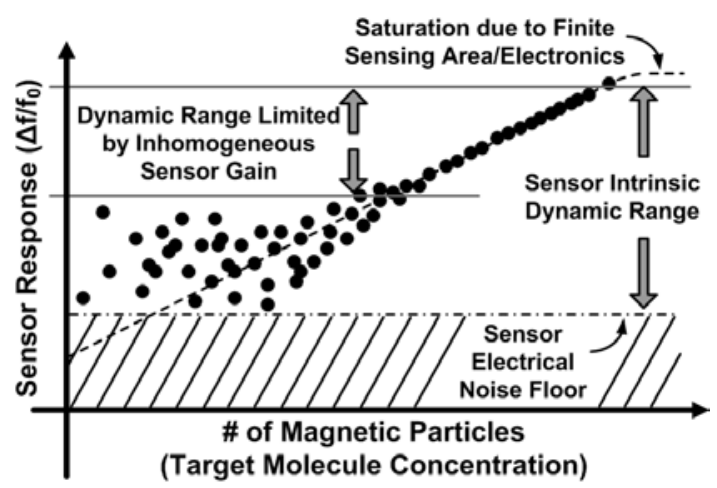

Fig. 2. Degraded dynamic range due to non-uniform sensor gain.

In order to address this issue, in the following sub-sections a new sensing inductor design methodology will be proposed to achieve a uniform sensor transducer gain.

\section{A. Conventional Symmetric Inductor}

Symmetric inductors are widely used for on-chip differential LC oscillators (Fig 3.a). However, a symmetric inductor typically presents a highly non-uniform magnetic field strength $|\mathrm{B}|$ on its surface. Measured radially from the inductor's center to its edge, the field strength first increases due to the closer distance towards the metal traces. Then $|\mathrm{B}|$ gradually achieves its peak value when the magnetic field addition from all the turns is maximized. The field strength decreases after this peak, because of the weaker coupling and greater distance from the traces. With this field distribution, generally only the center of a symmetric inductor presents a relatively uniform transducer gain, which significantly limits the linear sensing area.

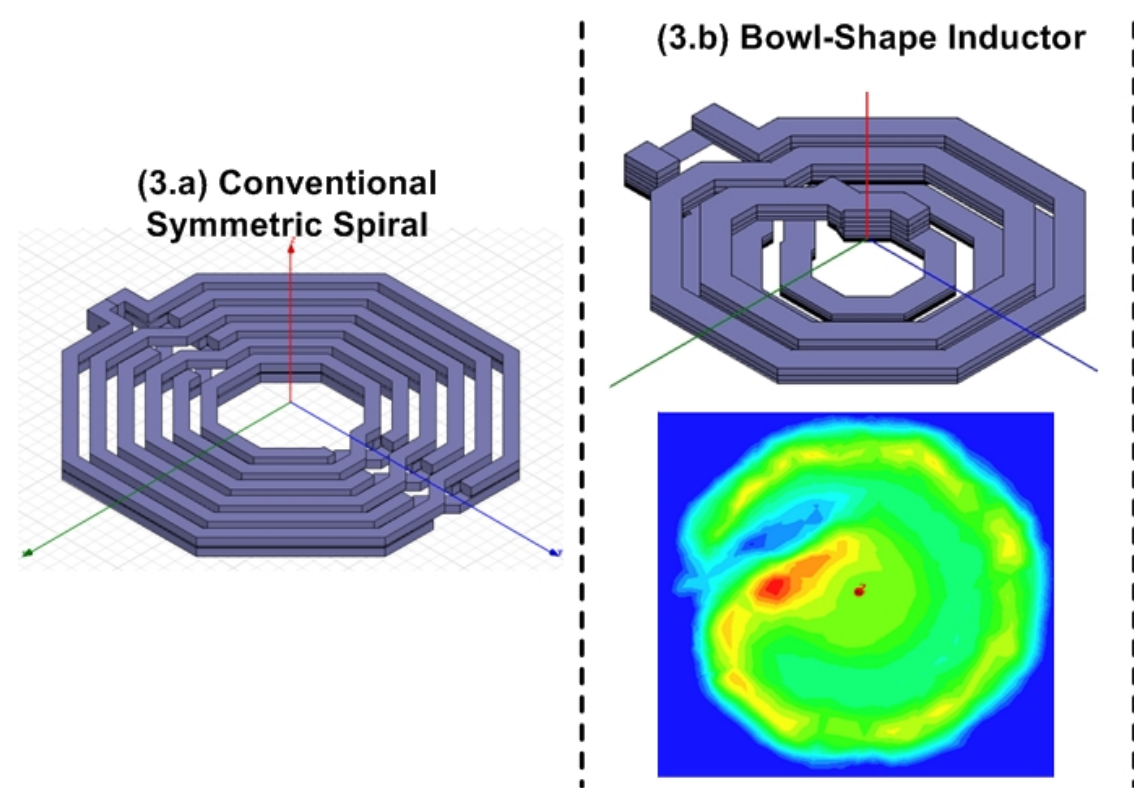

\section{(3.c) Bowl-Shape Inductor with Shimming Metal}
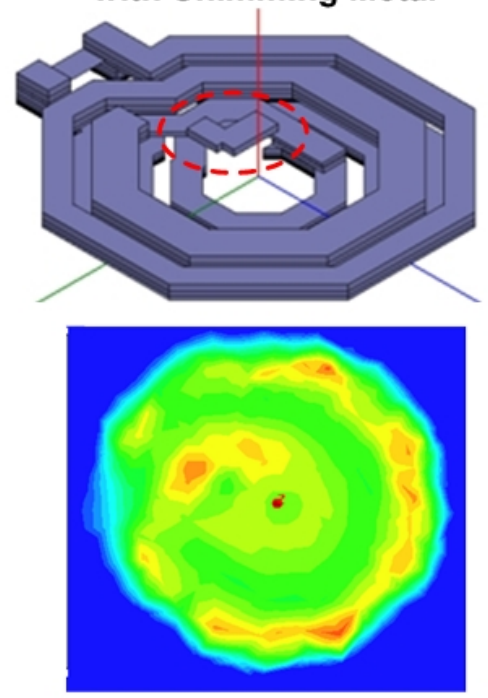

Fig. 3. Proposed sensing inductor design for uniform sensor gain. The normalized magnetic field strength $|\mathrm{B}|$ is plotted to compare the spatial uniformity. 


\section{B. Proposed Bowl-Shape Inductor}

Stacked coils are used to provide more degrees of freedom for shaping the polarization magnetic field. A dual-layer stacked inductor is proposed in Fig. 3.b, whose lower-level traces are deliberately spaced with respect to the upper ones to mitigate the $|\mathrm{B}|$ peaks and equalizes the magnetic field strength across the inductor. A significant uniformity on $|\mathrm{B}|$ can be observed. However, peaks and valleys of the field strength $|\mathrm{B}|$ exist at the connections between the two coil layers due to the current crowding effect.

\section{Floating Shimming Metal}

In order to suppress this non-uniformity, the interconnecting trace is designed to have a more gradual vertical transition between the layers to adjust the current distribution. Furthermore, a floating shimming metal structure is proposed as a parallel design option. The magnetic field of the floating metal induced by its eddy current changes the local total magnetic field strength and suppresses spatial $|\mathrm{B}|$ variation (Fig. 3.c). At $1 \mathrm{GHz}$ operating frequency, the simulated effective inductance and quality factor for the inductor show negligible changes after applying the shimming metal (Fig.4). Finally, the inner turn of the upper-layer trace is widened to further improve the transducer gain homogeneity.

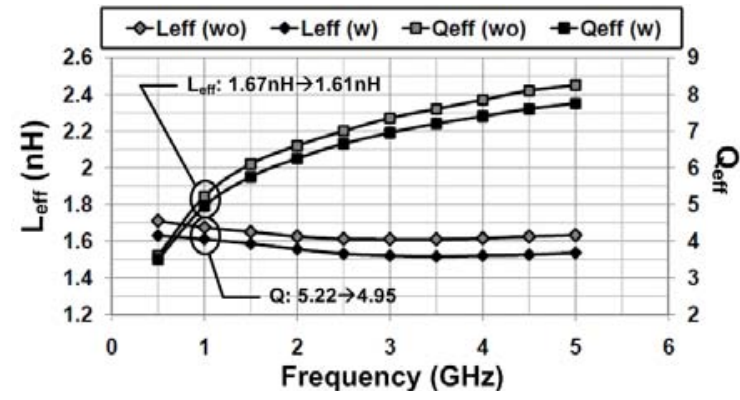

Fig.4. Inductance and Q with/without the floating shimming metal.

\section{SENSOR SYSTEM IMPLEMENTATION}

In this section, a design example implementing the proposed sensing inductor will be presented in detail.

Fig. 9 shows the schematic of the quad-core sensor cell with four LC tanks as the four sensing sites. The outer diameter of the sensing inductor is $110 \mu \mathrm{m}$.

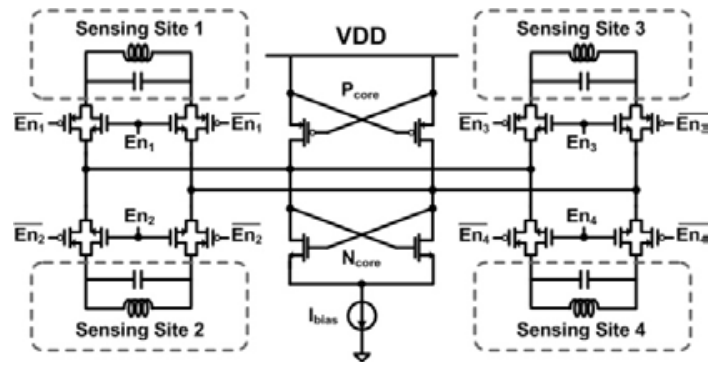

Fig. 5. Quad-core sensor schematic.

NMOS/PMOS switch pairs are used to couple the desired LC tank with the complementary active core. This sensor cell configuration provides differential sensing functionality by using any of the four sensing sites as a reference sensor and the other three as active sensors to suppress common-mode frequency-drift. Moreover, sharing the active core enables using Correlated Double Counting (CDC) for noise cancellation [5]. The oscillator's $1 / f^{3}$ phase noise due to active core flicker noise up-conversion generally limits the sensor noise floor. In the CDC scheme, this noise is correlated between differential sensing measurements through active core sharing and therefore receives direct suppression for sensitivity improvement.

A sensor array with 16 parallel sites is designed in a $45 \mathrm{~nm}$ CMOS SOI process (Fig. 6) at a total power consumption of $73 \mathrm{~mW}$. Multiplexers are used to feed the sensing oscillators' signals to the on-chip output buffers chain. The frequency results are eventually detected by an off-chip FPGA. This architecture is completely scalable to a very-large-scaled array on the same chip. In addition, since the IOs only have DC supply and digital signals, multiple chips can be easily tiled for ultra-high throughput applications, including genomic sequencing or genotyping. Fig. 7 shows the micro-photograph.

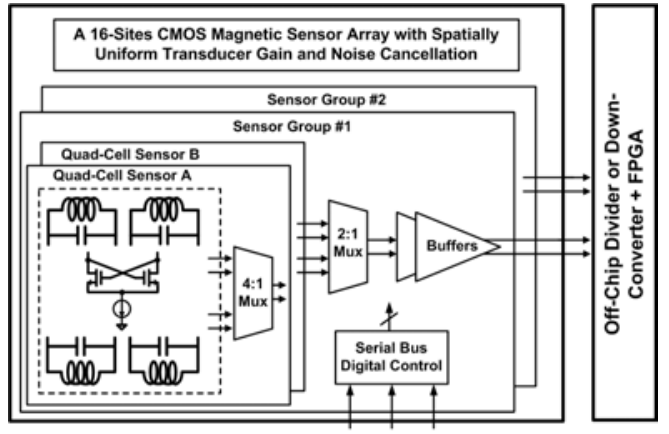

Data CLK Latch

Fig. 6. Sensor system architecture.

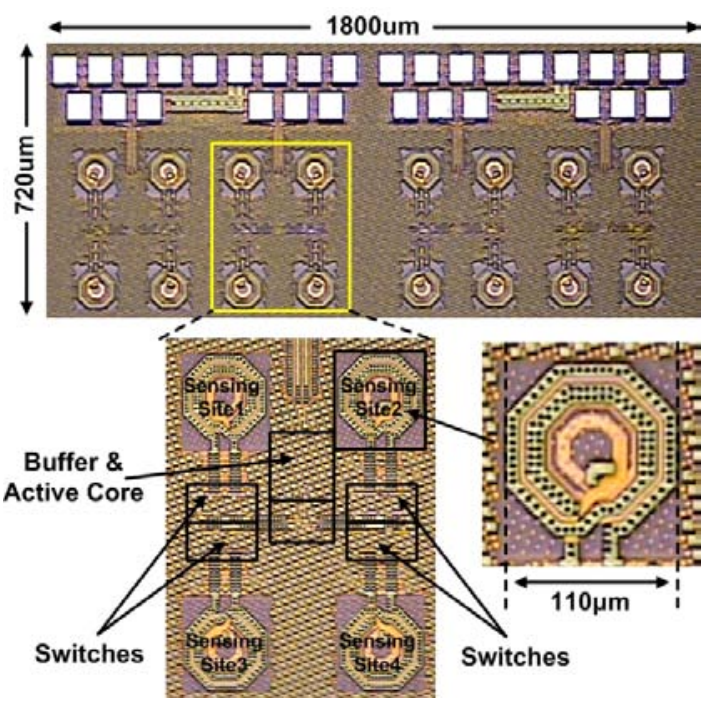

Fig.7. Chip microphotograph

\section{ELECTRICAL PERFORMANCE-NOISE CANCELLATION}

The sensing oscillator operates at a nominal frequency of $1.13 \mathrm{GHz}$. Its phase noise is measured with an RDL phase 
noise analyzer, achieving $-47.2 \mathrm{dBc} / \mathrm{Hz}$ and $-120.3 \mathrm{dBc} / \mathrm{Hz}$ at $1 \mathrm{kHz}$ and $1 \mathrm{MHz}$ offsets, respectively.

To verify the CDC noise cancellation functionality, the frequency counting results for different schemes (with counting duration $T$ of $0.1 \mathrm{~s}$ ) are shown in Fig.8. The normal differential scheme suppresses the common-mode frequencydrift, while an additional noise reduction (from $\sigma=1179 \mathrm{~Hz}$ to $\sigma=391 \mathrm{~Hz}$ ) is achieved after enabling the CDC scheme.

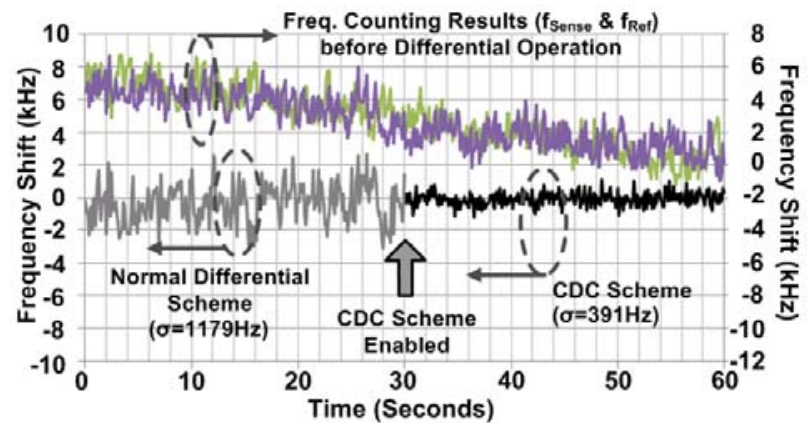

Fig.8. Frequency counting results in time domain for CDC scheme, normal differential and no differential operation.

During counting, the standard deviation of frequency measurement due to sensor electrical noise $\left(1 / \mathrm{f}^{3}\right.$ phase noise) is plotted with respect to different counting time $T$ in Fig.9. Overall, 10.6dB noise suppression is achieved.

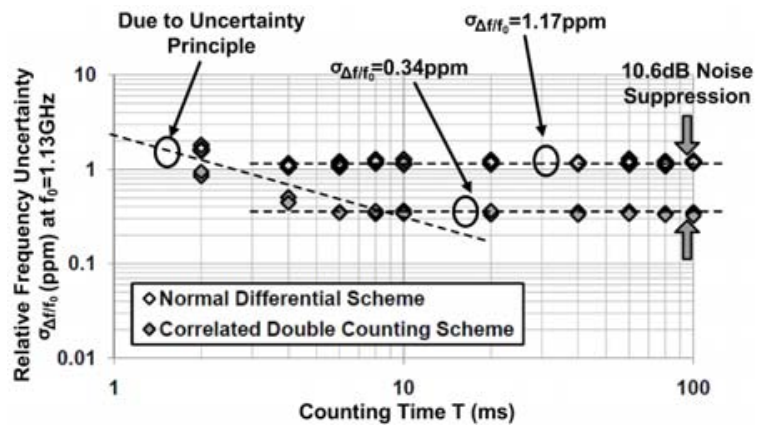

Fig.9. Frequency counting std. with different counting time $T$.

\section{MAGNETIC SENSING PERFORMANCE-UNIFORM TRANSDUCER GAIN}

To verify the sensor gain uniformity, two sets of magnetic sensing experiments are performed. Magnetic particles, DynaBeads ${ }^{\circledR}$ M450-Epoxy (Diameter $=4.5 \mu \mathrm{m}$ ), are used as the test samples in both measurements due to their ease of handling.

First, one single particle is randomly placed onto the sensing surface and the sensor responses together with the particles' positions are recorded and plotted in Fig.10. The consistent frequency-shift reading with an average value of $18 \mathrm{kHz}$ per particle verifies the uniform sensor transducer gain.

In the second experiment, different numbers of magnetic particles are deposited onto the sensor surface and their corresponding output frequency-shifts are shown in Fig.11. Note that with a noise floor of $388 \mathrm{~Hz}$ after CDC operation, a single $4.5 \mu \mathrm{m}$ magnetic particle is still far above our sensing limit. The measured linear response (up to 409 beads) indicates an effective dynamic range of at least $85.4 \mathrm{~dB}$. To the best of the authors' knowledge, this is the highest dynamic range among any CMOS biosensor modalities reported so far. Experiments with bio-samples on genomics level (DNA/RNA) and cellular level (bacteria) are currently under testing, whose results will be reported in the near future.

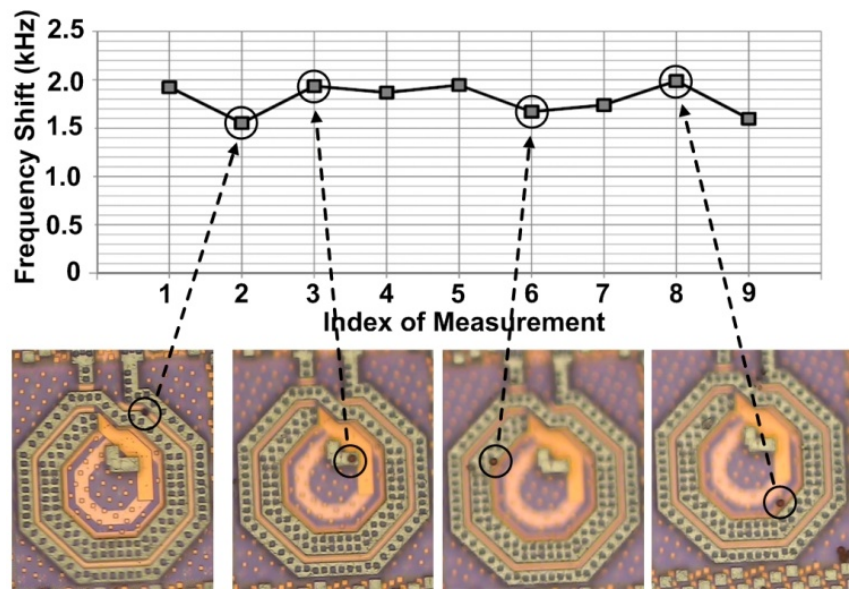

Fig.10. Response to a single randomly placed $4.5 \mu \mathrm{m}$ magnetic particle.

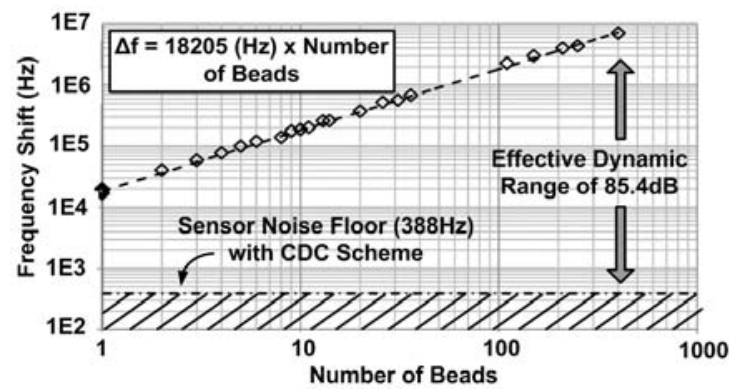

Fig.11. Sensor dynamic range for different particle numbers.

\section{CONCLUSION}

In this paper, a scalable ultrasensitive CMOS magnetic sensor array is presented. A new sensing inductor design method is proposed which significantly improves the spatial uniformity of the transducer gain across the sensing area and directly increases the system dynamic range. A 16-cell sensor array is implemented in a $45 \mathrm{~nm}$ CMOS SOI process together with the CDC noise cancellation scheme. The measurements verify both the spatially uniform transducer gain and the noise suppression functionality.

\section{REFERENCES}

[1] S. Han, et al., "A High-Density Magnetoresistive Biosensor Array with Drift-Compensation Mechanism," IEEE ISSCC Dig. Tech. Papers, pp.168-169, Feb. 2007.

[2] Y. Liu, et al., "CMOS Mini Nuclear Magnetic Resonance Systemand its Application for Biomolecular Sensing," IEEE ISSCC Dig. Tech. Papers, pp.140-141, Feb. 2008.

[3] P. Besse, et al., "Detection of A Single Magnetic Microbead Using a Miniaturized Silicon Hall Sensor," Appl. Phys.Letters, Vol 80, Issue 22, pp. 4199-4201, Jun. 2002.

[4] H. Wang, et al., "A Frequency-Shift CMOS Magnetic Biosensor Array with Single-Bead Sensitivity and No External Magnet," IEEE ISSCC Dig. Tech. Papers, pp.438-439, Feb. 2009.

[5] H. Wang, et al. "An Ultrasensitive CMOS Magnetic Biosensor Array with Correlated Double Counting Noise Suppression," to appear in IEEE MTT-S Int. Microwave Symp, May. 2010. 